\title{
Implementation of Wavelet Denoising and Image Morphology on Welding Image for Estimating HAZ and Welding Defects
}

\author{
Dibya Prakash Jena ${ }^{1}$, Rajesh Kumar ${ }^{2}$ \\ ${ }^{1}$ Manufacturing Management, BITS Pilani, Rajasthan, India, jena.dibya@gmail.com \\ ${ }^{2}$ Precision Metrology Laboratory, Department of Mechanical Engineering \\ Sant Longowal Institute of Engineering and Technology, Longowal - 148106, India, rajesh_krs@rediffmail.com
}

\begin{abstract}
In the current work, a filtering scheme for measurement of area and perimeter of heat affected zone (HAZ) in welding has been proposed along with identification of defects like porosity in the fusion zone. A filtering scheme based on wavelet filtering, edge detection and morphology has been designed and implemented on a welding image for this purpose. To see the effectiveness of the wavelet based proposed filtering scheme, SNR is calculated and compared with the filtered image processed without wavelet denoising. Experimental results revel that the SNR improves by $45-65 \%$ when wavelet filtering is introduced in the filtering scheme. Crisscrosses of the boundary of HAZ were observed and compared. The wavelet based proposed filtering scheme enhances the HAZ boundary smoothness by $12.5 \%$ in comparison to the filtering scheme without using wavelet denoising. In the limiting case, for poor quality image of weldment, the deviation in measurement of HAZ by manual and proposed scheme is within $10 \%$. Error in manual evaluation significantly contributes in the above deviation as the HAZ on the base metal side in the raw image is diffused and difficult to measure accurately.
\end{abstract}

Keywords: wavelet, edge detection, morphology, thresholding, heat affected zone (HAZ), porosity, signal to noise ratio

\section{INTRODUCTION}

$\mathrm{H}$ EAT AFFECTED ZONE (HAZ) and porosity in fusion zone are among some of the important parameters that influence quality of the weldment. While welding steel components, the fusion zone represents both the deposited metal and the part of the base metal (steel) melted during the process, and is a solidification macrostructure. The HAZ represents those regions in the close proximity of the weld, where the heat input during the welding process and subsequent re-cooling changes the microstructure without melting the base metal. The extent and magnitude of property change depends primarily on the base material, its thermal diffusivity, the weld filler metal, and the amount and concentration of heat input by the welding process. Development of surface defects and micro cracks is another issue in the welding process. Therefore, measurement of area of HAZ, and surface defect has been of great use in determining weld quality. In general, the heat affected zone is measured by conventional visual inspection techniques. But it is difficult to calculate the exact area of the HAZ and surface defects by manual stereological methods and conventional visual inspection techniques as the area to be evaluated is of irregular shape. Its approximation to any standard shape may lead to error in measurement. Also this method of evaluation is time consuming. In such a situation, analysis based on image processing can be more appropriate. In real image of a weldment, boundary of HAZ is diffused and not clear due to poor image quality. Locating edges at the correct position without any discontinuity is an important requirement for developing a suitable edge detection technique. Methods based on wavelet transform, fuzzy logic, etc., have been proposed by many researchers to detect the edge of the image corrupted by noise [1-6]. In isolation, so far, these techniques are not very much effective in identifying HAZ and surface defect precisely.
Standard edging filter has also been used for estimation of HAZ [7]. But the main difficulty is that the boundary of HAZ obtained by the method is very zigzag which may not be the true condition.

In the present work an improved filtering scheme is proposed with combination of wavelet denoising, gray scale thresholding, image morphology and a suitably designed boundary detection technique. It results in smoothness across HAZ and defects such as porosity in the image. It also helps in locating their boundaries precisely. Quantitative evaluation of area of HAZ and surface defects in a weldment is presented.

\section{IMAGE PROCESSING ALGORITHM}

The imaging sensor assigns a coordinate and a gray level or color value to each pixel that specifies the brightness or color of the pixel. An image is a $2 \mathrm{D}$ array of values representing light intensity $\mathrm{f}(\mathrm{x}, \mathrm{y})$ where $\mathrm{f}$ is the brightness of the point $(\mathrm{x}, \mathrm{y})$, and $\mathrm{x}$ and $\mathrm{y}$ represent the spatial coordinates of a picture element, or pixel. To identify the HAZ and surface defects in the specimen, we have implemented an image processing scheme/algorithm as shown in Fig.1. The raw image has high level of noise. To improve the SNR, wavelet based filtering scheme was implemented. In the first stage of the filtering scheme, wavelet denoising was implemented on the raw image. Further median filtering was implemented to remove the impulsive noise introduced in the image. In the second stage, hard thresholding technique was implemented on median filtered image to extract the HAZ and surface defects, which can be treated as objects. The thresholding value may differ from sample to sample, which depends upon the contrast of the object in the image. The thresholded image carries a lot of small holes and small particles inside and outside the HAZ object. In the next stage, to remove 
small particles, filling holes, close the object, morphological operations were implemented on the image. The object extracted after morphological operation was correlated with HAZ in weldment. Furthermore, an edge detection method was developed and implemented to detect the boundary of the HAZ.

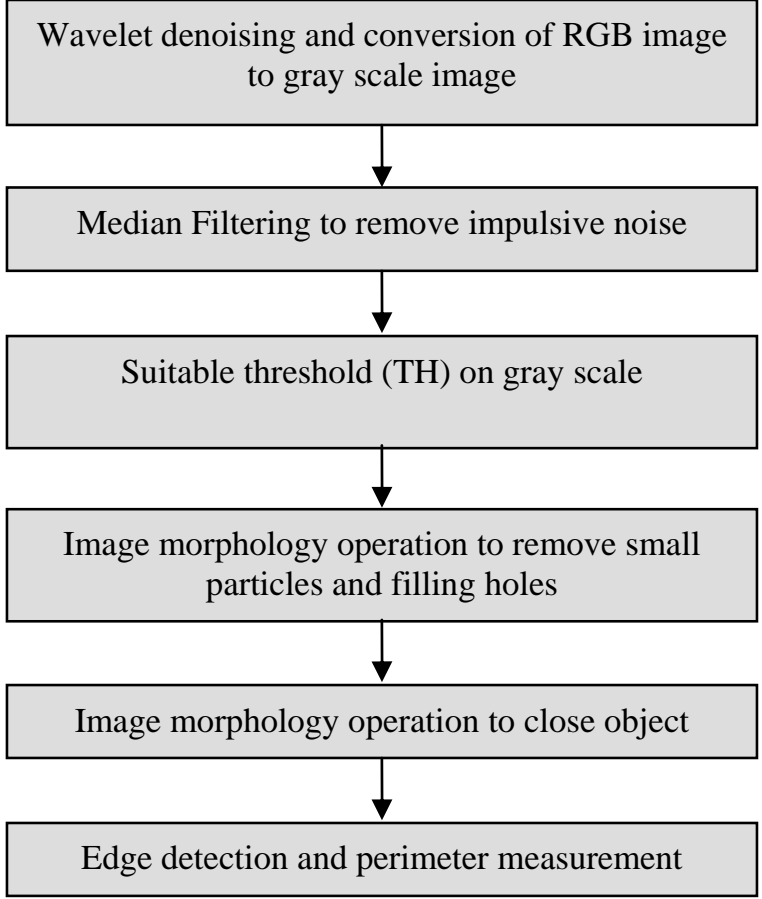

Fig.1. Flowchart of filtering scheme

Theoretical descriptions of each tool used in the algorithm are available in literature [7-9]. For denoising purpose Symlet-8 wavelet was used in this work, which allows the symmetric extension of data at the image boundaries and prevents discontinuities by a periodic wrapping of the data $[4,10,11]$. The wavelet thresholding procedure removes noise by thresholding the coefficient of the detail sub-bands, while keeping the low resolution coefficients unaltered. For smoothening purpose the median filtering was used to modify the image corrupted by impulsive noise. To remove small particles and filling holes, the morphological operations were used with suitable matrix size and element connectivity [7-9].

A filter was also developed and implemented for finding out the edge and perimeter of a particle in the image. The pixel value $P_{(x, y)}$ is compared $(\neq)$ with four neighborhood elements and concluded as boundary element [5]. Mathematically, this can be expressed as in Eq. (1). The boundary element $\left(P_{(x, y)}=0\right)$ is counted and interpreted as boundary lines with number of pixels. The total numbers of boundary pixels were converted in terms of length of the boundary line. Area in the closed boundary in terms of pixels is the estimate of area under consideration.

$$
\begin{aligned}
& {\left[\begin{array}{ccc}
P_{(x-1, y+1)} & P_{(x, y+1)} \neq P_{(x, y)} & P_{(x+1, y+1)} \\
P_{(x-1, y)} \neq P_{(x, y)} & P_{(x, y)} & P_{(x+1, y)} \neq P_{(x, y)} \\
P_{(x-1, y-1)} & P_{(x, y-1)} \neq P_{(x, y)} & P_{(x+1, y-1)}
\end{array}\right]} \\
& =\left[\begin{array}{ccc}
P_{(x-1, y+1)} & P_{(x, y+1)} & P_{(x+1, y+1)} \\
P_{(x-1, y)} & P_{(x, y)}=0 & P_{(x+1, y)} \\
P_{(x-1, y-1)} & P_{(x, y-1)} & P_{(x+1, y-1)}
\end{array}\right]
\end{aligned}
$$

\section{EXPERIMENT AND RESULT}

Test specimens of MAG welding were prepared by laying the beads along the length of the mild steel plate. These beads were laid at the welding parameter 30 volts, $210 \mathrm{amp}$ and wire feed rate $7 \mathrm{~mm} / \mathrm{min}$. The specimen was cut in perpendicular to welding speed direction in such a way that metallurgical changes in the direction of thickness due to welding could be examined. The polishing was done with emery paper according to the ascending order of the grit number and then etching using Nital solution (98\% methanol \& 2\% nitric acid). The image of the weldment after surface preparation was acquired with the help of CCD camera and IMAQ PCI-1409 image acquisition card. A typical raw image on gray scale of the specimen at the specified welding parameter is shown in Fig.2. The dark zone in the image just below the weld metal zone and above the unaffected parent metal zone in the figure is the HAZ. From the figure it is evident that at the base material side, the HAZ is defused.

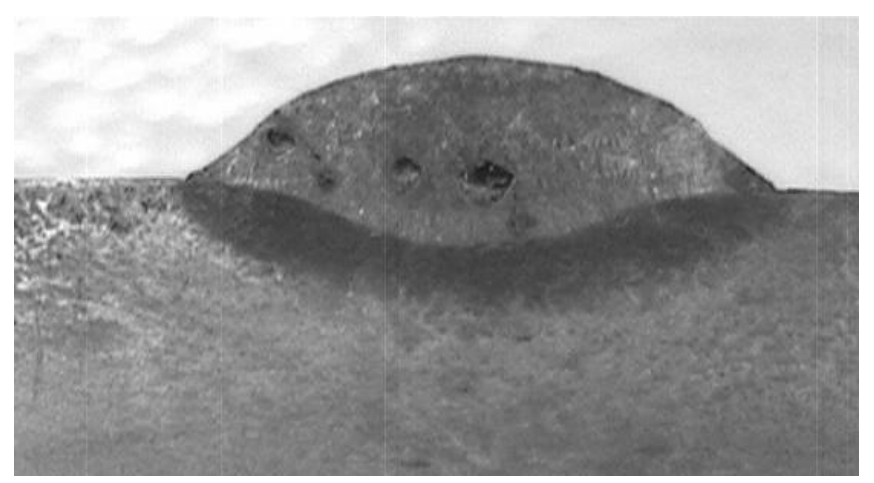

Fig.2. Raw image of the specimen

The images were processed for evaluation of area of HAZ. To calculate the area of HAZ and defects, millimeter was calibrated in terms of pixels $(1 \mathrm{~mm}=30$ pixels $)$ by putting a scale while acquiring the image.

\section{A. Filtering of image for evaluation of area of $\mathrm{HAZ}$}

Due to poor signal to noise ratio (SNR) of image, boundary of HAZ obtained after implementing the previously developed filtering schemes is more zigzag and differs from the actual one.

First of all, the area of HAZ is calculated without wavelet denoising but with processing through the other steps mentioned in the proposed filtering scheme. In this the gray threshold value 91 was used. The HAZ extracted in this fashion is projected over raw image and is shown in Fig.3, so that deviation in filtered HAZ area if any over the actual 
HAZ area can be visualized. The observed area of HAZ was $20.1 \mathrm{~mm}^{2}$ and the perimeter of HAZ was $39.5 \mathrm{~mm}$ respectively.

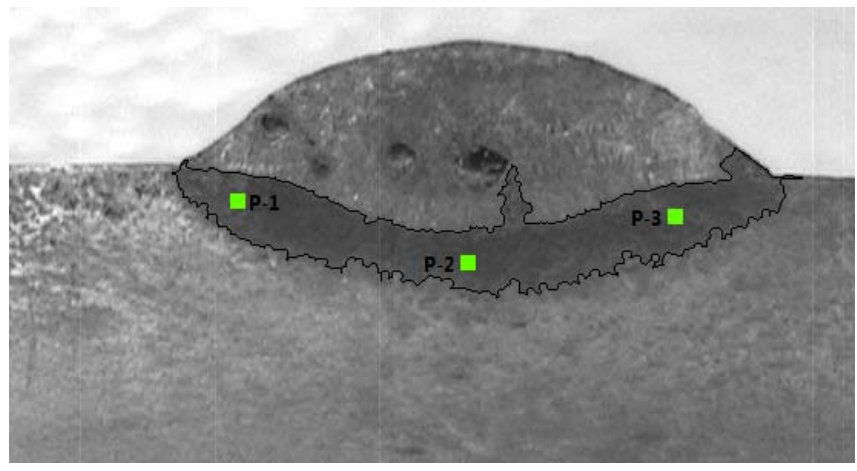

Fig.3. Extracted HAZ without wavelet denoising overlaped on raw image

Although the HAZ became smooth after filtering, the boundary of HAZ appears zigzag. To get improvement on this aspect the wavelet filtering was implemented on raw image and processed in accordance with the proposed filtering scheme. During wavelet denoising, the 2D discrete or un-decimated wavelet transform was implemented. The raw image was decomposed using Symlet- 8 wavelet up to level 3 with multiple rescaling methods. Sure thresholding technique was implemented and reconstructed. The HAZ filtered by the process is superimposed on the raw image and is shown in Fig.4. It was observed that apart from smoothness in the HAZ, its boundary also became smooth.

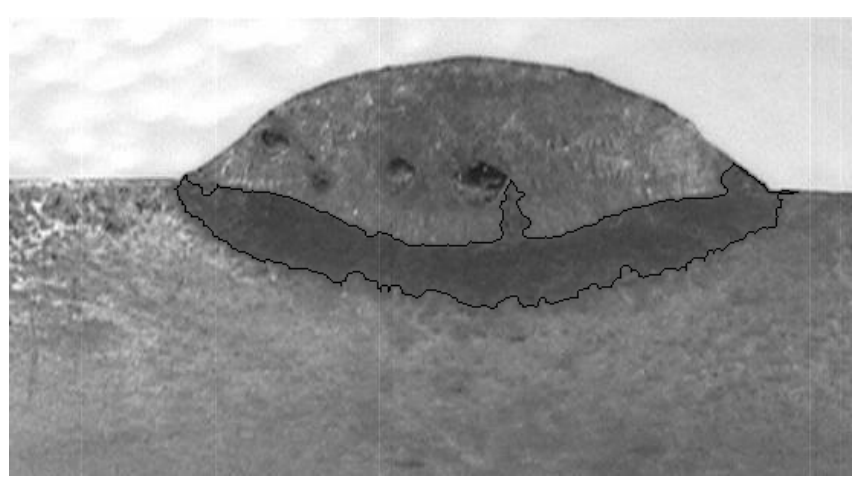

Fig.4. Extracted HAZ obtained after implementing the filtering scheme with wavelet denoising, overlaped on raw image

To see the effectiveness of the wavelet based filtering scheme mentioned in Fig.1, in comparison to the without wavelet preprocessed filtered image, SNR which is the quantitative performance indicator of smoothness of the image is calculated and compared, while observing the continuity and crisscrosses of the boundary of HAZ was observed qualitatively. The SNR was calculated for 10x10 matrixes at three locations P-1, P-2, P-3 on HAZ as marked in Fig.3. At the same positions for image in Fig.4 SNR is calculated. The values of SNRs are shown in Table 1. It was observed that the SNR improves by $45-65 \%$ after wavelet filtering.
Table 1. SNR calculated at three locations on HAZ

\begin{tabular}{|l|l|l|l|}
\hline Image & SNR at P-1 & SNR at P-2 & SNR at P-3 \\
\hline Fig.3 & 13.62 & 15.42 & 21.23 \\
\hline Fig.4 & 25.57 & 22.27 & 33.89 \\
\hline
\end{tabular}

The value of the area of HAZ and perimeter for filtered HAZs without and with wavelet preprocessing is shown in Table 2.

Table 2. Area of HAZ measured using image processing

\begin{tabular}{|l|c|c|l|l|}
\hline Image & $\begin{array}{l}\text { Pixels } \\
\text { in HAZ }\end{array}$ & $\begin{array}{l}\text { HAZ area } \\
\text { in } \mathrm{mm}^{2}\end{array}$ & $\begin{array}{l}\text { No. of } \\
\text { Pixels on } \\
\text { Perimeter }\end{array}$ & $\begin{array}{l}\text { Perimeter } \\
(\mathrm{mm})\end{array}$ \\
\hline Fig.3 & 18975 & 20.01 & 1185 & 39.50 \\
\hline Fig.4 & 18930 & 19.95 & 1040 & 34.67 \\
\hline
\end{tabular}

From the above table and Fig. 3 and 4, it is observed that due to introduction of wavelet denoising in the filtering scheme there is decrease in area of measured HAZ, i.e., loss of some data but at the same time smoothness of boundary of HAZ is much better than the smoothness of the HAZ appearing in Fig.3. The boundary of HAZ obtained by the proposed filtering scheme is much closer to the actual HAZ. Manual calculation of HAZ can be only an approximate one because HAZ on the base metal side is diffused.

\section{B. Additional Defect Information in the weldment}

A separate morphological operation was implemented on wavelet filtered image to identify defects. The wavelet filtered image was thresholded at gray scale 73 and large objects were removed using image morphology for 7 iterations with square or hexagonal element of connectivity 4-8 followed by morphological operations filling holes of connectivity 4-8 and proper close operation respectively.

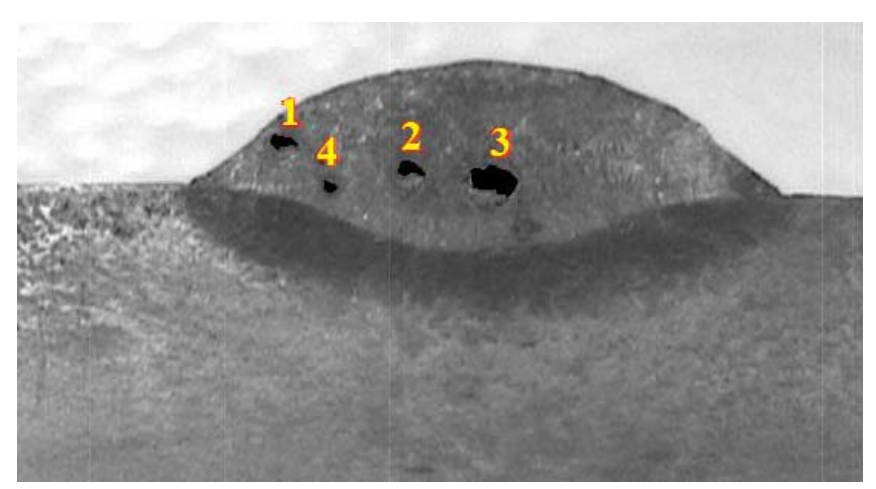

Fig.5. Defect identified and overlapped on raw image

Table 3. Defect size measurement

\begin{tabular}{|l|l|l|l|l|}
\hline $\begin{array}{l}\text { Defect } \\
\text { measurements }\end{array}$ & $\mathrm{D}_{1}$ & $\mathrm{D}_{2}$ & $\mathrm{D}_{3}$ & $\mathrm{D}_{4}$ \\
\hline $\begin{array}{l}\text { No. of pixels in } \\
\text { defect }\end{array}$ & 104 & 119 & 420 & 48 \\
\hline $\begin{array}{l}\text { Calculated value } \\
\text { of defect area } \\
\text { in } \mathrm{mm}^{2}\end{array}$ & 0.116 & 0.132 & 0.467 & 0.053 \\
\hline
\end{tabular}


The above process identifies six particles over the image. Further particle filtering technique was implemented in weld deposition zone. Finally, we identified 4 particles as shown in Fig. 5 in the weld deposition zone. The particle properties, for defect ' $\mathrm{D}_{1-4}$ ' are tabulated and shown in Table 3.

\section{Scope of Further Improvement}

The major difficulties on the proposed filtering scheme are setting proper threshold values and selecting appropriate mother wavelet function with level of decomposition. The threshold value will depend on image texture. Improper wavelet and level of decomposition selection may tend to blur the image which may loose required information to be measured. The fusion along with weld deposition measurement can be done with an image overlapped with HAZ boundary. Further improvements can be done to find an algorithm to get threshold value automatically and adaptive wavelet design to get a more smoother HAZ boundary.

\section{CONCLUSION}

The processing scheme based on morphology, thresholding and edge detection gives enhanced image and is very effective in identifying HAZ and welding porosity defects. Experimental results revel that the wavelet based proposed filtering scheme enhances the HAZ boundary smoothness by $12.5 \%$ in comparison to the without using wavelet denoising in the filtering scheme. In the limiting case, for poor quality image of weldment, the deviation in measurement of HAZ by manual and proposed scheme is within $10 \%$. Error in manual evaluation has significant contribution in the above deviation as the HAZ on the base metal side is diffused and difficult to calculate accurately. The filtering scheme is able to maintain correct location of the edges and hence precisely evaluates the area of HAZ without shifting. The image processing based measurement of the above mentioned welding parameters reduces manual effort and accuracy in shape although its boundary is complex and ambiguous.

\section{REFERENCES}

[1] Bhuiyan, M.I.H., Ahmad, M.O., Swamy, M.N.S. (2007). Spatially adaptive wavelet-based method using the Cauchy prior for denoising the SAR images. IEEE Transactions on Circuits and Systems for Video Technology, 17 (4), 500-507.

[2] Jena, D.P., Garanayak, H., Sahni, V., Kumar, R. (2010). Automatic grain counting in metal structure using image morphology and skeleton by zone of influence. International Journal of Applied Engineering Research, 5 (17), 2965-2973.

[3] Kumar, R., Jena, D.P. (2009). Phase determination in low carbon steel metallographic image using wavelet transform. Asian Journal of Chemistry, 21 (10), S125-129.

[4] Kumar, R., Shakher, C. (2004). Application of digital speckle pattern interferometry and wavelet transform in measurement of transverse vibrations in square plate. Optics and Lasers in Engineering, 42, 585-602.

[5] Sonka, M., Hlavac, V., Boyle, R. (2003). Image Processing, Analysis, and Machine Vision. Singapore: Thomson Asia Pte Ltd.

[6] Angrisani, L., Capriglione, D., Ferrigno, L., Miele, G. (2011). A new digital signal processing method for spectrum interference monitoring. Measurement Science Review, 11 (1), 1-8.

[7] Biswal, V., Soni, S., Jena, D.P., Kumar, R. (2008). Application of image processing in estimation of area of heat affected zone. Journal of Metallurgy and Materials Science, 50 (4), 219-225.

[8] Kizrak, A.M., Özen, F. (2011). A new median filter based fingerprint recognition algorithm. Procedia Computer Science, 3, 859-865.

[9] Hsueh, Y.-C. (1992). Mathematical morphology on limages. Signal Processing, 26, 221241.

[10] Daubechies, I. (1992). Ten Lectures on Wavelets. Philadelphia: Society For Industrial And Applied Mathematics.

[11] Mallat, A. (2009). A Wavelet Tour of Signal Processing. Academic Press. 